\title{
Klinische Diagnosen als soziale Konstruktionen
}

\author{
Volkmar Aderhold
}

Psychotherapie-Wissenschaft 8 (1) 25-32 2018

www.psychotherapie-wissenschaft.info

CC BY-NC-ND

DOI: $10.30820 / 8242.05$

\begin{abstract}
Zusammenfassung: Diagnosemanuale der zurückliegenden Jahrzehnte bewirkten oder beabsichtigten eine fortschreitende De-Kontextualisierung psychischer Störungen, die mit einer fortschreitenden Biologisierung der Psychiatrie einherging, die letzten Endes eher der Pharmaindustrie als den PatientInnen dient. Dabei ist die Befundlage der biologischen Psychiatrie alles andere als ermutigend, insbesondere auch deshalb, weil die diagnostischen Kategorien zu wenig valide sind. Das neue System der Research Domain Criteria (RDoC), das von funktionellen neuronalen Teilsystemen des gesunden Gehirns ausgeht, könnte die Neurobiologie weiterbringen. Ob dies gelingt, ist nicht absehbar.

Klinische Diagnosen dagegen sollten in erster Linie dem Verstehen und der Verständigung dienen. Da sie grundsätzlich Ergebnis eines sozialen Konstruktionsprozesses sind, sollte ein dialogisches Ko-Konstruieren mit den bedeutungsvollen Anderen in der sozialen Welt des Betroffenen im Zentrum stehen. Notwendig und unhintergehbar ist dabei eine innere und äussere Polyphonie dieser Wirklichkeitskonstruktionen. Psychotherapeutisches Expertenwissen ist Teil dieser Polyphonie.
\end{abstract}

Schlüsselwörter: Psychiatrische Diagnosen, ICD, DSM, Komorbidität, De-Kontextualisierung, Biologisierung, Research Domain Criteria (RDoC), Diagnostik als dialogischer Prozess, Polyphonie

\section{Was "sind" psychische Erkrankungen? Zweifel an der Validität psychiatrischer Diagnosen}

Psychische Erkrankungen und ihre diagnostische Einteilung sind eine gesellschaftliche und fachgesellschaftliche Konstruktion. Die Symptome, die als Kriterien für die Klassifikation dienen, sind als Phänomene dimensional in einer Population verteilt, das heisst die Grenze zwischen Normbereich («Normalität») und Abweichung («psychische Erkrankung») ist eine willkürliche, auf Konventionen beruhende Setzung. Diagnosen geben also psychischen Phänomenen, die ausserhalb einer statistischen Norm liegen, eine scheinbar kategoriale Ordnung, werden jedoch allein durch das Clustern von Phänomenen hergestellt und gestellt. Diese Cluster berücksichtigen keinerlei Ursachen, gleich welcher Art, also weder neurobiologische und biologische Ausgangslagen noch Kontextfaktoren noch kulturelle Bedingungen oder interaktionelle Faktoren. Die Phänomene verlieren ihren Reaktionscharakter und werden so vereigenschaftlicht. Erkrankungen «brechen aus» oder «exazerbieren» gemäss einer vermeintlich biologischen Gesetzmässigkeit. Als psychisch krank bezeichnete Menschen werden in der Folge verdinglichter wahrgenommen und behandelt.

Psychische Erkrankungen zu definieren wird in modernen Gesellschaften an Expertinnen und Experten delegiert. Symptomzentrierte Diagnosen sind Ergebnisse eines Rituals, das als notwendig angesehen wird, um als medizinische Fachdisziplin anerkannt zu werden. Und so nehmen Psychiaterinnen und Psychiater einen ebenbürtigen Rang neben ihren medizinischen Kolleginnen und Kollegen ein. Und auf diese Weise behalten sie auch weitgehend die Kontrolle über ein Fachgebiet, für das auch andere Disziplinen, allen voran die Psychologie und Psychotherapie, «Herrschaftsanspruch» erheben könnten.

Ein grosses Ziel des ICD-10 und DSM-IV und ihrer Weiterentwicklung war es Mitte der 1990er Jahre, die Diagnosepraxis international und vor allem zwischen wissenschaftlichen Studien vergleichbar zu machen. Das führte zwar national und international zu einer besseren Zuverlässigkeit (Reliabilität) von Diagnosen in wissenschaftlichen klinischen Studien. In Feldstudien zur Verwendung des DSM-5 in der Alltagspraxis war die Reliabilität der Diagnosen jedoch durchgängig schwach, oftmals nicht besser als mit den alten Diagnosesystemen (vgl. hierzu Freedman et al., 2013). Die Fortentwicklung der Diagnostischen Manuale ging sogar mit gravierenden Fehlentwicklungen einher, wie im Folgenden ausgeführt wird.

Kulturelle Differenzen wurden durch die Diagnosemanuale nivelliert. Dass indigene Sichtweisen und Erklärungen verdrängt wurden und sich die Pharmaindustrie weitere Märkte erschliessen konnte, wird als Kolonisierung gewertet, die für die Bevölkerung der neu «erschlossenen» Länder oftmals mehr Nachteile als Vorteile hervorbringt (vgl. Summerfield et al., 2008; Watters, 2009).

«Komorbidität» wird suggeriert, indem frühere, sinnvolle Regeln des Diagnostizierens ausser Kraft gesetzt wurden. Zu den Fehlentwicklungen gehören: die Abkehr von der Hierarchie- und Schichtenregel bei Überlappung diagnostischer Kategorien; falsche Interpretation diagnostischer Begriffe aufgrund fehlender oder unpräziser Definitionen; der sogenannte polythetische Ansatz, demgemäss lediglich ein Mindestmass an diagnostischen Kriterien für 
die Diagnose erfüllt sein muss; unscharfe Abgrenzung zwischen Zusatzsymptomen und komorbiden Störungen; Nichtberücksichtigung von Ausschlusskriterien; Einbezug sukzessiv (Längsschnitt) und simultan (Querschnitt) auftretender Symptome. Der so entstehende Eindruck, dass es hohe Raten an «Komorbiditäten» gebe, steht im Widerspruch dazu, dass die Diagnosen keinesfalls eigenständige Grunderkrankungen abbilden.

Eine reine Querschnittsbetrachtung der Symptomatik führt ausserdem zu häufigen Diagnosewechseln, wenn Symptome hinzutreten oder wegfallen oder sich auch nur der Bericht der Patientinnen und Patienten über die Symptome ändert.

Die schon früher bemängelte Beobachterabhängigkeit der Diagnosen wurde also nicht reduziert, sondern durch die scheinbar gewachsene Objektivität nur stärker verschleiert. Sie wird unter Umständen noch verstärkt durch mangelhafte psychopathologische Kompetenz, subjektive Vorlieben, Gegenübertragungsphänomene, aber auch strategische Überlegungen. Hierunter fallen sowohl das betrügerische «Upgraden» aus ökonomischem Interesse als auch das «Downgraden» zur Stigma-Minimierung. Diagnosen dienen auch dazu, psychiatrische Leistungen abzurechnen. Was die Validität der Diagnosen betrifft, so zeigen sich Leistungsträger weitgehend unbekümmert.

Mit der Einführung des DSM-5 ging die strukturelle Validität der diagnostischen Konstrukte noch weiter verloren. Was sich als eigenständige Krankheitskategorie eigentlich hinter einem Cluster verbirgt, bleibt unzugänglich oder verliert sich in der weiterhin grossen Heterogenität innerhalb der Syndrome. Dies wird als beabsichtigte und vorteilhafte «Ätiologieunabhängigkeit» dargestellt.

Daher hat Thomas Insel, seinerzeit Direktor des $\mathrm{Na}$ tional Institute of Mental Health (NIMH), bereits zwei Wochen vor der Veröffentlichung des DSM-5 angekündigt, dass das NIMH dem Manual die Unterstützung entziehe und keine Forschung finanzieren werde, die ausschliesslich auf DSM-5-Diagnosen beruhe. Er begründete dies mit der mangelhaften Validität des DSM-5 und des darin enthaltenen Sammelsuriums an Symptombeschreibungen. Die Patientinnen und Patienten hätten etwas Besseres verdient. Er verglich diese Art psychiatrischer Diagnosestellung mit einer Praxis in der Somatik, bei der Beschreibung unterschiedlicher Arten von Brustschmerz ohne eine Zuordnung zu den Ursachen als ausreichende Diagnosen verwendet würden (vgl. Insel, 2013).

Die Veränderungen der diagnostischen Kategorien und der Kriterien innerhalb der Cluster sind, über die verschiedenen Versionen der Diagnose-Manuale hinweg, gross. Auch der Versuch des DSM-5, Diagnosen neu zu ordnen, macht dies erneut deutlich.

Die Diagnose «Schizophrenie» zeigt besonders deutlich, wie willkürlich die Ordnungsversuche der Diagnosemanuale sind. Historisch liegt zum Beispiel die Schnittmenge der verwendeten Symptomcluster für Schizophrenie seit ihrer Erfindung durch Bleuler (1911) bis 1984 bei 27 Prozent, das heisst über 70 Prozent der Menschen, die in einem System so definiert werden, würden in einem anderen Diagnosesystem diese Diagnose nicht erhalten (vgl. Katschnig, 1984). Für Betroffene ist jedoch von lebensgeschichtlich entscheidender Bedeutung, ob sie diese Diagnose erhalten oder nicht.

Gerade wurden mit dem DSM-5 auch die Untergruppen der Schizophrenie nach Bleuler (1911) - hebephren, kataton, paranoid und undifferenziert - wegen geringer Stabilität, Reliabilität und prognostischer Relevanz abgeschafft. Ebenso werden die Symptome ersten Ranges von Kurt Schneider nicht mehr als Kriterien angewendet. Schneider hatte diese ursprünglich (1938) zwar nur heuristisch angenommen, sie galten jedoch über Jahrzehnte als "pathognomonisch", das heisst für eine sichere Diagnosestellung ausreichend. Nach über 70 Jahren wurden sie nun doch nicht empirisch bestätigt. Späte Empirie für eine angeblich evidenzbasierte wissenschaftliche Diagnostik!

Zur Diagnose einer Schizophrenie reichen im ICD-10 und wohl auch im kommenden ICD-11 vier Wochen Symptomdauer aus, im DSM sind es hingegen sechs Monate. Das ist ein gravierender Unterschied, der auch nach drei Revisionen zwischen den Expertengruppen bestehen bleibt. Dies belegt erneut, wie willkürlich Entscheidungen getroffen werden - Entscheidungen, die gleichwohl gravierende Folgen für viele Klientinnen und Klienten haben.

\section{Interessengeleitetes Vorgehen}

Fachgesellschaften berufen sich bei der Definition diagnostischer Einheiten zwar auf wissenschaftliche Evidenz, tatsächlich erfolgt die Entscheidungsfindung jedoch durch Mehrheitsabstimmungen - und diese sind interessengeleitet. Denn die Mehrheit der Abstimmenden partizipiert an dem lukrativen Pharmalobbyismus mit bis zu siebenstelligen jährlichen Honoraren. Viele machen geltend, sie seien unbeeinflussbar, da sie Honorare von mehreren Firmen bezögen.

Die meisten Cluster, die psychische Abweichung bezeichnen, unterliegen kulturellen Normen und werden seit Jahrzehnten von den Vermarktungsinteressen der Pharmaindustrie beeinflusst.

So sorgten zum Beispiel Initiativen der Pharmaindustrie in Japan dafür, dass zuvor als nicht krankhaft erlebte leichtere Depressionen nach der Einführung der Antidepressiva vom Typ SSRI für die Verordnung der SSRI zugänglich wurden. Dass bei leichten und mittelschweren Syndromen die Antidepressiva den Placebos so gut wie nicht überlegen sind $\left(\mathrm{NNT}^{1}=10\right)$ und diese Syndrome auch ohne Medikation remittieren, konnte erst später in Metaanalysen nachgewiesen werden, indem aufgrund des «freedom of information act» (US-Congress 2005) auch unpublizierte Studien mit negativen Ergebnissen ausgewertet werden konnten:

1 NNT: Number needed to treat [dt.: Anzahl der notwendigen Behandlungen]. Die NNT ist eine statistische Masszahl, die angibt, wie viele Patienten pro Zeiteinheit (zum Beispiel ein Jahr) mit der Testsubstanz oder Testmethode behandelt werden müssen, um das gewünschte Therapieziel bei einem Patienten zu erreichen. 
«In einer Metaanalyse, in der Studien mit direkten Vergleichen zwischen offener Psychotherapie und offener Pharmakotherapie depressiver Episoden ausgewertet wurden, fand sich eine signifikante, in Bezug auf die absolute Patientenzahl jedoch geringe Überlegenheit von Antidepressiva gegenüber Psychotherapie (NNT = 14) (Cuipers et al., 2015), sodass die Autoren selbst zum Schluss kommen, dass die hier identifizierten Unterschiede wohl nicht als klinisch relevant betrachtet werden können» (S3 NVL Unipolare Depression, Version 3, 2015, S. 19).

Geradezu irreführend ist daher folgende Therapieempfehlung: Die aktuelle Versorgungsleitlinie für Depressionen (2016) fasst im Abschnitt «Pharmakotherapie» mittelschwere und schwere Depression einfach zusammen (vgl. ebd., S. 66). Bei mittelschweren Depressionen, bei denen es kaum Wirkvorteile der Pharmakotherapie im Vergleich mit Placebo $(\mathrm{NNT}=10)$ gibt, sollen so offenbar ebenfalls möglichst viele Betroffene «leitliniengerecht» mit Antidepressiva behandeln werden.

In Deutschland werden immer häufiger SSRI bei depressiven Syndromen verschrieben, ohne dass zum Beispiel jemals eine verkürzte Arbeitsunfähigkeit nach Pharmakotherapie bei den Krankenkassen zu verzeichnen war. Da die Verordnungen von Antidepressiva in Deutschland in den zurückliegenden 22 Jahren um 680 Prozent zugenommen haben und es auch in letzten neun Jahren zu einer weiteren Verdopplung gekommen ist, müssen auch immer mehr natürliche Reaktionen auf Lebenserfahrungen und medikalisiert worden sein, denn eine Zunahme der Schweren Psychischen Erkrankungen (SMI) lässt sich wissenschaftlich nicht belegen (vgl. Richter et al., 2008).

So gaben in einer Umfrage von 1683 antidepressiv behandelter Menschen 65 Prozent an, zuvor ein oder mehrere belastende Ereignisse oder Umstände erlebt zu haben wie Beziehungsprobleme (19\%), Lebensübergänge $(19 \%)$, Verluste (18\%), Probleme am Arbeitsplatz (15\%), andere gesundheitliche Probleme $(15 \%)$ und seltener soziale Isolation, Missbrauch, Gewalt, finanzielle Probleme (vgl. Hartdegen et al., 2017). Dies ist ein Beispiel dafür, wie de-kontextualisierende diagnostische Kategorien einen direkten und schwer korrigierbaren Weg in die Pharmakotherapie ebnen und - im Interesse der Pharmaindustrie - auch ebnen sollen.

Die Wirksamkeit von Antidepressiva war in der Untersuchung mit der Annahme biogenetischer Ursachen der eigenen Beschwerden positiv korreliert.

Die Vorstellung eines (bis heute wissenschaftlich nicht belegten) chemischen Ungleichgewichts als Ursache der Beschwerden korrelierte wiederum mit der Qualität der Beziehung zu dem verschreibenden Arzt (vgl. Read et al., 2015).

Kombiniert man demnach Antidepressiva mit Psychotherapie muss sich es in vielen Fällen - zumindest implizit - eine Konkurrenz der Erklärungsmodelle und beteiligten Professionellen einstellen. Da Antidepressiva ja weitgehend über Placebo-Effekte und damit Attribution wirken, benötigen sie dafür die Übernahme einer biologischen Erklärung. Dies muss notwendig viele psychothera- peutische Wirkfaktoren schwächen. Kann eine wirksame Psychotherapie auch den Wirkfaktor Placebo schwächen?

Die Medikalisierung setzt Patientinnen und Patienten auch nicht voraussagbaren Nebenwirkungen aus. Je länger die Einnahme, desto mehr und stärker. In der zitierten Befragung nehmen sie mehr als die Hälfte länger als drei Jahre ein. Die häufigsten Nebenwirkungen waren sexuelle Schwierigkeiten (62\%), emotionale Betäubung $(60 \%)$, das Gefühl nicht man selbst zu sein $(52 \%)$, eine Abnahme der positiven Gefühle (42\%), weniger Fürsorge für andere $(39 \%)$, Suizidalität (39\%) und Entzugserscheinungen $(55 \%)$, die komplettes Absetzen oft verunmöglichen (vgl. Read et al., 2014). Dies sind offensichtlich auch Nebenwirkungen, die einem Gelingen von Psychotherapie entgegenstehen.

Weitere Anstiege der Verschreibungen gelingen sogar auch mit den Neuroleptika - und das trotz vieler alarmierender Befunde über ihre schädigenden Nebenwirkungen. Zudem zeigen neuere Studien eine deutlich geringere nachweisbare Wirkung mit einer Erhöhung der NNT von früher 3 bis 4 auf heute 6 .

Steigerungen der Medikamenten-Verordnungen sind ebenfalls bei der generalisierten Angststörung, dem ADHSyndrom, der Bipolaren Störung im Kindes- und Jugendalter zu verzeichnen - und demnächst vermutlich auch bei Trauerreaktionen, die, halten sie länger als vier Wochen an, ebenfalls als pathologisch gewertet werden, sodass eine antidepressive Therapie empfohlen wird.

\section{De-Kontextualisierung und Biologisierung psychischer Phänomene}

Die gute Absicht und der gute Zweck, die Diagnosestellung unabhängig von der theoretischen Orientierung des Diagnostikers zu machen, hat sich ins Gegenteil verkehrt: Mit den Theorien sind auch die psychosozialen Kontexte aus dem Blick geraten. Und obwohl heutige Diagnosen scheinbar ursachenunabhängig gestellt werden, führt die Diagnosestellung oft zu einer impliziten und expliziten Biologisierung und «Genetisierung» der syndromalen Konstrukte. Beides hat Nachteile. Diese De-Kontextualisierung führt häufig dazu, dass die Diagnose für die betroffenen Menschen ungünstige bzw. die falschen Konsequenzen hat. Denn die Biologisierung bahnt zu oft den Weg in primäre oder sogar ausschliessliche Pharmakotherapie. Nachfolgend gehe ich auf die Nachteile im Einzelnen ein.

\section{Primat der Pharmakotherapie}

Die Biologisierung dient vor allem der Pharmakologisierung der psychiatrischen Behandlung. Syndrome werden so definiert, dass sie spezifischen pharmakologischen Interventionen zugänglich werden. Scham etwa wird durch die generalisierte Angststörung den Antidepressiva zugänglich, «aufsässiges» Verhalten von Kindern durch die bipolare Störung den Antipsychotika, eine längere 
Trauerreaktion den Antidepressiva. Die Gattungsnamen der Psychopharmaka suggerieren eine diagnosespezifische heilsame Wirkung. Oft wird - völlig irreführend - eine Analogie zur Diabetesbehandlung mit Insulin hergestellt:

«Ein Psychose-Kranker in der akuten Situation hat ein Problem mit dem Gehirnbotenstoff Dopamin. Diese Menschen brauchen dann ein Medikament, genauso wie ein Patient mit zu hohem Blutzucker Insulin braucht. Durch Reden geht das Problem nicht weg» (Falkai, 2013).

\section{Angebliche «Nicht-Verstehbarkeit» von Verhalten}

Je stärker diejenigen Gesellschaften, die internationale Diagnosen verwenden, von den (Post-)Industriegesellschaften abweichen, umso weniger kulturell kompatibel sind diese Diagnosen. Zudem entfremden sie von anderen gesellschaftlichen/ethnischen Verstehens- und Handlungskonzepten. Bekannt ist diesbezüglich des schamanischen Konzepts eines «initial call», das phänomenologisch oftmals einer ersten psychotischen Episode entspricht, und der Besessenheit von Geistern, die mit einem Ritual für den Betroffenen und seine Familie oder die Dorfgemeinschaft beantwortet wird.

\section{Prognose}

Diagnosen erlauben keine auch nur annähernd sichere Prognose. Von Gesundung bis Chronifizierung ist meist alles möglich. Trotzdem werden Diagnosen oft zu einer selbsterfüllenden Prophezeiung, indem sie meist negative Zukunftsvorstellungen bei den Betroffenen und Angehörigen aber auch Professionellen bewirken.

\section{Stigmatisierung}

Diagnosen haben oft stigmatisierenden, seltener entlastenden Charakter. Der Versuch, das Ausmass der Stigmatisierung psychischer «Erkrankungen» durch ihre Biologisierung zu mindern, ist vermutlich gescheitert. Mit der gesellschaftlichen Expansion von Diagnosen mit erfundenen biologischen Erklärungsmodellen nimmt die soziale Stigmatisierung eher zu, nicht ab. Hirnkranke erfahren stärkere soziale Ablehnung als Menschen, die durch negative Lebenserfahrungen in psychische Krisen geraten sind. Sie scheinen uns fremder, in ihren Handlungen unverständlicher und unkontrollierbarer - und sich selbst ebenfalls (vgl. Angermeyer et al., 2014). Diagnosen, die eine medizinisch-humanistische Schutzfunktion vor Überforderung und Zurückweisung ausüben sollten, verkehren sich so in ihr Gegenteil.

Placebostudien und Tierforschung legen jedoch die hohe Bedeutung von positiven Erwartungen und Hoffnung für neuroplastische Prozesse auch und gerade unter Psychopharmaka nahe. Psychopharmaka können unter negativen Erwartungsbedingungen eine ebenfalls negative Wirkung haben (Nocebo-Effekt, vgl. Rief et al., 2016).

\section{Biologische Argumente gegen die Biologisierung}

Diagnosen lassen sich bis heute nicht durch eindeutige biologische Phänomene (Biomarker) absichern. Es gibt zum Beispiel bis heute - also auch nach 40 Jahren Bildgebung - keine konsistenten und verlässlichen anatomischen und funktionellen Veränderungen, die sich eindeutig den klinischen Beobachtungen bei Menschen mit Schizophrenie-Diagnose zuordnen lassen oder die zu Veränderungen in der Behandlung geführt hätten (vgl. hierzu Fusar-Poli et al., 2016, S. 2011). Der Nachweis, dass es sich bei der Schizophrenie um eine Erkrankung des Gehirns handele, konnte also bis heute nicht erbracht werden. Griesingers Diktum «Geisteskrankheiten sind Hirnerkrankungen» sollte dringend ad acta gelegt werden. Auch für Depressionen wurden bis heute keine brauchbaren neurobiologischen Erklärungen gefunden. Die «chemical imbalance»-Theorie ist mittlerweile obsolet. Auch wenn sich neurobiologische Substrate vereinzelt finden lassen, sind sie fast immer nur ein Zwischenglied in einer langen Transmissionskette mit unklarem Beginn. So ist zum Beispiel regional erhöhtes Dopamin bei psychotischen Phänomenen sicher nicht die «Ursache» von Psychosen, sondern nur die gemeinsame "pathophysiologische Endstrecke» (Howes et al., 2009) diverser anderer, mit Stress und Trauma verbundener Lebensbedingungen (vgl. Varese et al., 2012), deren mögliche genetische Komponente bis heute ungeklärt ist (vgl. Johnson et al., 2017).

Viele psychische Phänomene lassen sich als Symptome mehreren unterschiedlichen Krankheitskategorien zuordnen, ebenso gibt es kaum genetische oder neurobiologische Befunde, die nicht mehreren Kategorien zugeordnet werden können. Psychische Phänomene lassen sich also keineswegs durch genetische oder neurobiologische Befunde erklären. Beide Phänomenbereiche sind voneinander relativ unabhängig:

«Looking for the neurochemistry of mental disorders that don't necessarily exist has turned out to be as futile as using a map of the moon to get around Manhattan» [«Die Suche nach der Neurochemie von psychischen Störungen, die nicht unbedingt existieren, hat sich als so sinnlos erwiesen, wie sich mit einer Karte des Mondes in Manhattan zu orientieren»] (Greenberg, 2013; Übersetzung V.A.).

Zum biologischen Determinismus gesellt sich oft noch ein mehr oder weniger ausgeprägter genetischer Determinismus hinzu. Dabei ist heute erwiesen, dass Gen-UmweltInteraktionen weit komplexer sind als es die immer noch verbreitete 50/50 Prozent Zuschreibung zu Gen und Umwelt glauben machen. Genetische Faktoren werden weit häufiger durch Umwelterfahrungen kontrolliert (Epigenetik). Die Verheissungen waren vor 20 Jahren gross, aber echte therapierelevante Fortschritte in der Genetik 
sind bis heute nicht zu verzeichnen. Die Genetiker selbst schreiben die Stagnation in der Forschung der Tatsache zu, dass bis heute keine validen und ausreichend homogenen diagnostischen Konstrukte existieren, das heisst auch innerhalb einer diagnostischen Kategorie die Heterogenität zu gross ist. Die genetischen Determinanten werden bei Menschen mit «Schizophrenie-Diagnose» heute in individuell unterschiedlichen polygenetischen Clustern und einzelnen nukleotiden Polymorphismen (SNP) vermutet, die jedoch jeweils nur minimale Effektstärken aufweisen. Genetische sowie neurobiologische Befunde treffen entweder auf mehrere diagnostische Kategorien oder nur auf Subsyndrome zu. Andere diagnostische Kategorien sind nicht in Sicht.

\section{Aktuelle Krise der Psychiatrie}

Der Krug geht so lange zum Brunnen, bis er ...

Die Psychiatrie hat sich in eine Situation hineinmanövriert, die von grosser Verunsicherung und Instabilität gekennzeichnet ist. Das Projekt Neurobiologisierung der Psychiatrie hat bisher keine Ergebnisse von tragfähiger Evidenz erbringen können: Diagnostik, Genetik, ursächliche biologische Mechanismen, kausal wirkende Pharmakotherapie, ausreichende Effekte der Pharmakotherapie, Verminderung der Chronifizierungstendenz, wirksame pharmakotherapeutische Frühinterventionen gibt es nicht. Nur aus Symptomen lassen sich keine validen Syndrome ableiten, die durch spezifische neurobiologische Prozesse determiniert werden. Andererseits lassen sich gegenwärtig aus den neurobiologischen Befunden keine konsistenten diagnostischen Klassifikationen ableiten. Diese Lücke soll durch neue basale diagnostische Kriterien geschlossen werden.

Als basale Kategorien sollen sogenannte Research Domain Criteria (RDoC) gelten. Sie stellen den Beginn eines neuen Ordnungssystems für die Grundlagenwissenschaft dar. Dieses System ist vollkommen unbeeinflusst von bestehenden diagnostischen Kategorien, sondern geht von den neuronalen Systemen des gesunden Gehirns aus. Die RDoC sind:

1. Negatives Valenzsystem (aversive Motivation/Angst)

2. Positives Valenzsystem

3. Kognitives System

4. System für soziale Prozesse

5. Arousal/Regulatorisches System

Den RDoC werden dann genetische, bildgebende, physiologische und kognitive Daten zugeordnet, um weitere Erkenntnisse aus dieser Zusammenschau zu gewinnen. Diese Daten werden jedoch nicht mehr durch Diagnosen abgegrenzt bzw. ausgegrenzt. Diese Cluster sollen dann mit Symptomen und Behandlungseffekten in Beziehung gesetzt werden. Dieses Projekt wird viele Jahre bis Jahrzehnte dauern. $\mathrm{Ob}$ es erfolgreich sein wird, ist unklar. Schon jetzt werden von namhaften Wissenschaftlern erhebliche Zweifel geäussert (zum Beispiel von Fava, 2014). Das NIMH hingegen ist begeistert. Im Prinzip können die
Wissenschaftlerinnen und Wissenschaftler so weitermachen wie bisher, lediglich werden die Befunde verfeinert und man ordnet diese einander anders zu.

Grundsätzlich steht das Gehirn als soziales Organ im Gegensatz zu solchen Konzepten. Soziale Erfahrungen sind weitgehend bestimmend für kognitive, affektive und relationale Lern- und Entwicklungsprozesse. Im Hinblick auf die Ausprägung von normabweichenden psychischen Phänomenen sind weitere negative soziale Ereignisse und Prozesse von entscheidender Bedeutung. Wie auch immer die ererbte genetische Disposition sich darstellt, sie ist vermutlich nie hinreichend, um erhebliche normabweichende Syndrome zu determinieren. Es ist vermutlich eher umgekehrt: Bereits sehr frühe soziale Erfahrungen scheinen die Genomfunktion, zum Beispiel durch DNAMethylierung, rückwirkend zu modulieren.

Für Psychosen wurden zum Beispiel die folgenden sozialen Faktoren ermittelt und bereits repliziert: biologische und psychische Schwangerschaftskomplikationen, Geburtskomplikationen, frühe Verlusterfahrungen, frühe instabile Umwelten, elterliche Konflikte, sexuelle, physische und emotionale Traumatisierungen, Vernachlässigung, Aufwachsen in Städten, soziale Fragmentierung, soziale Notlagen, soziale Ablehnung und Niederlagen, Bullying, Diskriminierung, Migration, Cannabis.

Diese dekonstruktive Darstellung von «invaliden» Krankheitskonstruktionen soll ermutigen. Das brüchige Gebäude psychischer Erkrankungen wird nur noch notdürftig von einem Gerüst aus diagnostischen und pharmakologischen Gewohnheiten stabilisiert. Dieser Psychiatrie sollte man unverfroren(er) gegenübertreten. Längst hat der Kaiser keine Kleider mehr an. Psychotherapeutisches Handeln sollte sich keinesfalls mehr an diese Krankheitsund Diagnosekonstrukte binden und binden lassen.

Der neurobiologische Rückgriff auf die oben beschriebenen basalen Dimensionen der RDoC soll ermutigen, auch im Bereich psycho-sozial-spirituellen Verstehens auf sinnvolle, Orientierung gebende Verstehensansätze zurückzugreifen, die wir ja schon haben und die es weiterzuentwickeln gilt. Es wäre an der Zeit, dass sie aus ihrem Schattendasein treten und die Lücken, die neurobiologische Forschung bisher hinterlassen oder hergestellt hat, durch ihre verstehende und auch erklärende Potenz schliessen.

\section{Diagnosen als dialogische De- und Re-Konstruktion}

Diagnosen bleiben auch weiterhin historisch geprägte, das heisst Übergangsphänomene. Daran ändert auch der Umstand nichts, dass neurobiologisch orientierte Wissenschaftlerinnen und Wissenschaftler neue Systeme in der Erwartung konstruieren, mit ihnen als abweichend definierte psychische Phänomene später erklären zu können. Man darf gespannt und begründet skeptisch sein: Wie auch immer die Befunde korreliert und kategorisiert werden, man wird keine Ursachen, sondern allenfalls Begleitumstände, neuronale Korrelate oder Bindeglieder 
in den Entstehungsmechanismen psychischer Phänomene als Ausdruck lebendiger Selbstorganisation finden.

Nimmt man die immer komplexeren und immer stärker sozial determiniert erscheinenden neurowissenschaftlichen Befunde zu Bewusstsein, Selbst- und Wirklichkeitserleben ernst, wird man in den Erwartungen sehr viel bescheidener und vorsichtiger. Offensichtlich sind wir nicht mit subjektiven erlebten Wirklichkeiten, sondern nur mit inneren repräsentierenden Strukturen für die Welt und unserem Selbst im Kontakt. Einen direkten Kontakt durch Sinneswahrnehmungen mit einer Aussenwelt haben wir jedoch nicht. Es erscheint uns nur so, sogenannte phänomenale Repräsentationen im Sinne eines naiven Realismus (vgl. Metzinger, 2013).

Die RDoC sind also eine Anstrengung mit offenem Ausgang, die wohl recht sicher zehn Jahre und länger in Anspruch nehmen wird. Es ist nicht zu erwarten, dass bis dahin die gegenwärtigen Diagnosesysteme aufgegeben werden. Daher muss man einen strategischen Umgang mit ihnen finden, sie jedoch nicht allzu ernst nehmen. Dies lässt sich in der Regel mit den Betroffenen gut besprechen. Oft ist es sinnvoll, sie selbst zu fragen, welchen Umgang sie sich mit Diagnosen wünschen.

Klinische Diagnosen sollten dem Verstehen und der Verständigung dienen. Da sie grundsätzlich Zuschreibungen darstellen und eine starke Tendenz zur Identifikation, Verdinglichung, zur potenziellen Selbstentfremdung oder auch Zurückweisung sowie Aufrechterhaltung dieser Zuschreibung durch institutionelle, soziale und individuelle adaptive Prozesse in Gang setzen können, ist Vorsicht geboten. Dadurch, dass vielfältige belastende, pathogene, traumatisierende soziale Prozesse personal verortet werden und so vereigenschaftlicht werden, führen Diagnosen weiterhin zu einer hauptsächlich individuellen Attribuierung der bedingenden sozialen, kulturellen, gesellschaftlichen und ökonomischen Faktoren. Menschen neigen dazu, Fremdzuschreibungen in ihr eigenes Selbstbild zu übernehmen und sich demgemäss zu verhalten (vgl. Wiesner et al., 2016; Hacking, 2006). Wichtig und eine grosse Chance für Betroffene wäre es deshalb, psychische Phänomene durchgängig auf ihren relationalen Charakter hin zu untersuchen. Sie sind fast immer Ausdruck eines Beziehungsgeschehens. Aber auch diese Betrachtungsweise wäre noch zu schwach, um der intersubjektiven Konstruktion von Wirklichkeiten gerecht zu werden.

Jede psychiatrische Diagnose ist Ergebnis eines sozialen Konstruktionsprozesses, der keine physikalischen, nicht einmal biologische, sondern psychische und soziale Phänomene intersubjektiv konstruiert. Die Kontext- und Beobachterabhängigkeit psychischer Phänomene ist insofern konsequent zu berücksichtigen. Alles ist immer nur von einem bestimmten Beobachter gesagt und gilt nur in den untersuchten Kontexten. Diagnosen werden in der Sprache und den gegenwärtigen Denkmustern von Psychologie und Psychiatrie gestellt. Der Konstruktionsprozess dieser Diagnosen bezieht seine Berechtigung aus seiner therapeutischen Funktion. Im besten Falle führt der diagnostische Prozess zum Gefühl des Verstandenwerdens und Sich-selbst-Verstehens, im schlechtesten Falle zum Gefühl der Stigmatisierung und Diskriminierung.

Für das therapeutische Handeln müssen Benennungen von abweichend erlebten oder definierten psychischen Phänomenen hilfreich und nicht behindernd oder sogar destruktiv sein bzw. wirken. Damit sind an Diagnosen andere Anforderungen zu stellen. Sie sollten sich ihres historischen Übergangscharakters bewusst sein und damit nicht als wahr, sondern im besten Falle als nützlich aufgefasst werden. Sie sollten dem individuellen und intersubjektiven Verstehen und Verstandenwerden dienen, wobei der Andere nicht allein der professionelle Andere ist, sondern auch die lebensweltlich bedeutsamen Anderen einzubeziehen sind. Der Einzelne sollte als soziales Individuum in einem die subjektiven Wirklichkeiten und das Selbsterleben ko-konstruierenden Kontext verstanden werden. Dies wird durch das Addieren von Symptomen, die teilweise und zeitweise vorhanden sein müssen, unmöglich gemacht. Verstehen gelingt nur durch Individualisierung und nicht durch Klassifizierung und Kategorisierung.

Am Anfang des diagnostischen Prozesses steht die individuelle Beschreibung. Diese jedoch ist ein dialogisches Geschehen, bezieht also immer den Anderen mit ein, verändert sich durch den und die Anderen, allein aufgrund der Komplexität. Die infrage stehenden psychischen und sozialen Phänomene sind unscharf, vage, vieldeutig. Diese Intersubjektivierung und das dialogische Ko-Konstruieren in einem diagnostischen Prozess mit den bedeutungsvollen Anderen in der sozialen Welt des Betroffenen sollten statt professioneller Extraktion und Abstraktion im Vordergrund stehen. Notwendig und unhintergehbar ist dabei eine innere und äussere Polyphonie dieser Wirklichkeitskonstruktionen. Jeder sieht notwendig nur einen/seinen Teil, der andere sieht es notwendig anders als wir, und wir sehen es meist unterschiedlich zu unterschiedlichen Zeiten und sogar auch gleichzeitig. Psychiatrisches Expertenwissen ist Teil dieser Polyphonie. Der diagnostische Konstruktionsprozess wäre dementsprechend Teil der natürlichen Lebenswelt, vielperspektivisch und vieldimensional. Im dialogischen Prozess wäre dann Diagnostik und Therapie kaum mehr zu trennen. Prototyp eines dialogisch-diagnostisch-therapeutischen Prozesses wären Netzwerkgespräche der wichtigsten Protagonisten. In ihnen kommen vor:

$>$ subjektive und intersubjektive Phänomene,

$>$ Beschreibungen statt Definitionen, eher vage und mehrdeutig,

$>$ Kontextbedingungen,

$>$ Entwicklungsgeschichte, Entwicklungsbedingungen,

$>$ Rekonstruktion von Sinn und Bedeutung von bedeutsamen Lebenserfahrungen/-ereignissen,

$>$ Auswirkungen im Alltag und Lebensumfeld,

> Prozesshaftigkeit der Phänomene, zeitliche Limitierung, Episodik in der Sprache der Psychiatrie,

> Berücksichtigung der hohen natürlichen Adaptationsfähigkeit von Individuen und Systemen,

$>$ Ressourcen,

$>$ Begründungen und Bedingtheiten, 
$>$ intersubjektive Bedeutungen,

$>$ innere Penetranz der Phänomene (Wie stark habe ich sie? Bin ich sie?)

Psychische Krisen werden so in die Identität und das biografische Selbstverständnis re-kontextualisiert. Diagnosen werden dann Teil von Narrativen, eher im Sinne von Sprachbildern (Metaphern) anstatt von fixen Kategorien.

Diese Konstruktionen sollten prozessoffen und hermeneutisch vielfältig und unabschliessbar sein, um eine unangemessene Identifikation mit einer Konstruktion zu vermeiden. Vermutlich liessen sich durch ein solches Vorgehen viele Syndrome bereits im Prozess der Verständigung nach Monaten abschwächen oder weitgehend wieder auflösen.

$\mathrm{Ob}$ ein solcher verstehender, dialogischer, intersubjektiver Prozess Anschluss finden könnte an die neurobiologischen Konstruktionen, bleibt abzuwarten. Beide bleiben zwar grundsätzlich unterschiedliche Zugänge mit unterschiedlichen Reichweiten. In Anerkennung des jeweils anderen haben sowohl erklärende wie verstehende Zugänge auch auf unabsehbare Dauer ihre Berechtigung. Damit könnte sich die aktuelle Psychiatrie wieder heraustrauen in ein mehr suchendes, in ethischer Weise auch experimentelles Vorgehen in therapeutischen, verstehenden und erklärenden Zusammenhängen.

\section{Literatur}

Angermeyer, M.C., Millier, A., Kouki, M., Refaï, T., Schomerus, G., Toumi, M. (2014). Biogenetic explanations and emotional reactions to people with schizophrenia and major depressive disorder. Psychiatry Research, 220, 702-704. https://doi.org/10.1016/j.psychres.2014.07.038 (28.02.2018).

Bleuler, E. (2011). Dementia praecox oder Gruppe der Schizophrenien. https://www.psychosozial-verlag.de/pdfs/leseprobe/616.pdf (19.02.2018).

Cuijpers, P. \& Cristea, I. A. (2015). What if a placebo effect explained all the activity of depression treatments? World Psychiatry, 14, 310-311. https://doi.org/10.1002/wps.20249 (28.02.2018).

Cartwright, C., Gibson, K., Read, J., Cowan, O., Dehar, T. (2016). Long-term antidepressant use: patient perspectives of benefits and adverse effects. Patient Prefer Adherence, 10 (2016 Jul 28), 1401-7. https://www.ncbi.nlm.nih.gov/pmc/articles/PMC4970636/ (28.02.2018).

Falkai, P. (2013). Interview Apothekenumschau Januar. http://www. meinungsverbrechen.de/tag/dopamin-hypothese/ (31.01.2017).

Fava, G. A. (2014). Road to nowhere. World Psychiatry, 13, 49-50. https://doi.org/10.1002/wps.20108 (28.02.2018).

Freedman, R., Lewis, D.A., Michels, R., Pine, D.S., Schultz, S.K., Tamminga, C., Gabbard, G. O., Gau, S.S., Javitt, D. C., Oquendo, M., Shrout, P., Vieta, E. \& Yager, J. (2013). The initial field trials of DSM-5: New blooms and old thorns. American Journal of Psychiatry, 170, 1-5. https://doi.org/10.1176/appi.ajp.2012.12091189 (28.02.2018).

Fusar-Poli, P. \& Meyer-Lindenberg, A. (2016). Forty years of structural imaging in psychosis: promises and truth. Acta Psychiatrica Scandinavica, 134, 207-224. https://doi.org/10.1111/acps.12619 (28.02.2018).

Greenberg, G. (2013). The Rats of N.I.M.H. New Yorker (16.05.2013). http://www.newyorker.com/tech/elements/the-ratsof-n-i-m-h (16.01.2017).

Hacking, I. (2006). Making up people. Londo Review of Books, 28(16),
23-26. https://www.lrb.co.uk/v28/n16/ian-hacking/making-up-people (28.02.2018)

Hartdegen, M., Gibson, K., Cartwright, C. \& Read, J. (2017). Stressful events and circumstances reported by patients prior to being prescribed antidepressants. N Z Med J, 130(1448), 45-53. http:// europepmc.org/abstract/med/28081556 (28.02.2018).

Howes OD, Kapur S. (2009) The dopamine hypothesis of schizophrenia: version III- the final common pathway. Schizophr Bull., 35(3),549-562.

Insel, T. (2013). https://www.nimh.nih.gov/about/directors/thomasinsel/blog/2013/transforming-diagnosis.shtml (31.01.17).

Johnson EC, Border R, Melroy-Greif WE, de Leeuw CA, Ehringer MA, Keller MC (2017) No Evidence That Schizophrenia Candidate Genes Are More Associated With Schizophrenia Than Noncandidate Genes. Biol Psychiatry., 82(10),702-708.

Katschnig, H. (1984). Der «Polydiagnostische Ansatz» in der psychiatrischen Forschung. In A. Hopf \& H. Beckmann (Hrsg.), Forschungen zur biologischen Psychiatrie (S. 63-78). Berlin/Heidelberg/New York: Springer. https://doi.org/10.1007/978-3-64269539-1_8 (28.02.2018).

Metzinger, Th. (2013). Empirische Perspektiven aus Sicht der Selbstmodell-Theorie der Subjektivität: Eine Kurzdarstellung mit Beispielen. http://www.philosophie.uni-mainz.de/Dateien/Metzinger_ SMT_2013.pdf (16.01.2017).

Read, J., Cartwright, C. \& Gibson, K. (2014). Adverse emotional and interpersonal effects reported by 1829 New Zealanders while taking antidepressants. Psychiatry Res., 216(1), 67-73. http:// www.sciencedirect.com/science/article/pii/S0165178114000833 (28.02.2018).

Read, J., Cartwright, C., Gibson, K., Shiels, C. \& Magliano, L. (2015). Beliefs of people taking antidepressants about the causes of their own depression. J Affect Disord. 174, 150-6. http://www.sciencedirect.com/science/article/pii/S0165032714007101 (28.02.2018).

Richter, D., Berger, K. \& Reker, T. (2008). Nehmen psychische Störungen zu? Eine systematische Literaturübersicht. Psychiatr Prax. 35(7), 321-30. https://www.thieme-connect.com/products/ejournals/html/10.1055/s-2008-1067570 (28.02.2018).

Rief, W., Barsky, A.J., Bingel, U., Doering, B.K., Schwarting, R., Wöhr, M. \& Schweiger, U. (2016). Rethinking psychopharmacotherapy: The role of treatment context and brain plasticity in antidepressant and antipsychotic interventions. Neuroscience and Biobehavioral Review, 60, 51-64. https://doi.org/10.1016/j.neubiorev.2015.11.008 (28.02.2018).

S3. Nationale Versorgungsleitlinie Unipolare Depression, Version 3, 2015. https://www.dgppn.de/fileadmin/user_upload/_medien/download/pdf/kurzversion-leitlinien/S3-NVLdepressionlang_2015.pdf (12.01.2017).

Summerfield, D. (2008). How scientifically valid is the knowledge base of global mental health? British Medical Journal, 336, 992994. https://doi.org/10.1136/bmj.39513.441030.AD (28.02.2018).

Varese, P., Smeets, F., Drukker, M. et al. (2012). Childhood adversities increase the risk of psychosis: a meta-analysis of patient-control, prospective- and cross-sectional cohort studies. Schizophr. Bull., 38, 661-671.

Watters, E. (2009). Crazy like us: The Globalization of the American Psyche. New York: Free Press.

Wiesner, M., Epstein, E. \& Duda, L. (2016). SPRACHEMACHTSINN - Die Krise der Psychotherapie und der Weg zu einer posttherapeutischen Zukunft Systemmagazin 19. August 2016. http://systemagazin.com/sprachemachtsinn-die-krise-der-psychotherapie-und-der-weg-zu-einer-posttherapeutischen-zukunft/ (31.01.2017).

\section{Clinical diagnoses as social constructs}

Diagnostic manuals of recent decades intended or effected an ongoing de-contextualization of mental disorders, which went 
hand in hand with the advancing biologizing of psychiatry, that ultimately served the pharmaceutical industry rather than the patients. Moreover, the findings of biological psychiatry are far from encouraging, notably because the diagnostic categories lack validity. The Research Domain Criteria (RDoC) constitutes a new research framework that is based on functional neural subsystems of the healthy brain and may advance neurobiology. It is not foreseeable whether this approach will succeed.

The first and foremost objective of clinical diagnoses, on the other hand, should be understanding and communication. Since they are fundamentally the result of a social construction process, the primary focus should be on co-constructing through dialogue with the significant others in the social environment of the person concerned. Accordingly, an inner and outer polyphony of these constructions of reality is necessary and inevitable. Psychotherapeutic expertise also forms a part of this polyphony.

Keywords: Psychiatric Diagnoses, ICD, DSM, Comorbidity, De-contextualization, Biologizing, Research Domain Criteria (RDoC), Diagnosis as a Dialogue Process, Polyphony

\section{Diagnosi clinica come costruzione sociale}

I manuali di diagnosi degli ultimi decenni funzionano e propongono una progressiva decontestualizzazione dei disturbi psichici, la quale è andata insieme a una progressiva biologizzazione della psichiatria, che alla fine serve più l'industria farmaceutica dei pazienti. A riguardo la conoscenza scientifica della psichiatria è tutt'altro che incoraggiante, in particolare anche perché le categorie diagnostiche non sono sufficientemente valide. Il nuovo sistema di Research Domain Criteria
(RDoC), che deriva dai sottosistemi neuronali funzionali del cervello sano potrebbero far progredire la neurobiologia. Se ciò riesca, è da vedere.

Le diagnosi cliniche per contro devono servire prima di tutto alla comprensione e all'informazione. Poiché esse fondamentalmente sono il risultato di un processo di costruzione, al centro di esso dovrebbe trovarsi un dialogo di co-costruzione con gli altri attori significativi nel mondo sociale dei soggetti. È quindi necessaria e inevitabile una polifonia interna ed esterna di costruzioni di verità. Il sapere psicoterapeutico fa parte di questa polifonia.

Parole chiave: diagnosi psichiatriche, ICD, comorbidità, decontestualizzazione, biologizzazione, Research Domain Criteria (RDoC), diagnostica del processo dialogico, polifonia

\section{Der Autor}

Volkmar Aderhold, Dr. med., ist Arzt für Psychiatrie, Psychotherapie und Psychotherapeutische Medizin sowie Lehrender für Systemische Therapie und Beratung (DGSF). Seit 1982 arbeitet er in der Psychiatrie, davon 10 Jahre als Oberarzt im der Klinik für Psychiatrie und Psychotherapie des Universitätsklinikums Hamburg Eppendorf. Aktuell ist er Mitarbeiter der Instituts für Sozialpsychiatrie an der Universität Greifswald und wirkt durch Vorträge, Veröffentlichungen und die Beratung von psychiatrischen Organisationen im Bereich der Strukturentwicklung.

\section{Kontakt}

v.aderhold@gmx.de 